\title{
FATORES ASSOCIADOS À CRONOLOGIA DE ERUPÇÃO DE DENTES DECÍDUOS - REVISÃO DE LITERATURA:
}

\section{Erupção de dentes decíduos e fatores associados}

\author{
Maria Emília Queiroz DUARTE ${ }^{1}$ \\ Mayra Amaral ANDRADE ${ }^{2}$ \\ Patrícia Corrêa FARIA ${ }^{3}$ \\ Leandro Silva MARQUES ${ }^{4}$ \\ Maria Letícia Ramos JORGE 5
}

RESUMO: O objetivo desta revisão de literatura foi verificar os fatores que influenciam a cronologia de erupção dos dentes decíduos. A busca bibliográfica foi realizada na base de dados PubMed e Google Acadêmico, considerando-se o período de 1956 a dezembro de 2010. Utilizou-se como descritores "nutritional status and eruption of deciduous teeth", changes in the eruption of deciduous teeth", "malnutrition and eruption of deciduous teeth, premature birth and eruption of deciduous teeth". Adotou-se como limites: periódicos publicados na língua inglesa e do total de artigos encontrados ( $\mathrm{n}=1482$ ), 30 foram lidos na íntegra por estarem diretamente ligados ao tema. Observou-se na literatura consultada que a erupção dos dentes decíduos é influenciada por fatores como estado nutricional infantil, nascimento prematuro, tempo de amamentação, presença de síndromes, nível socioeconômico, gênero e condições maternas durante a gestação. A inter-relação entre estes fatores promove modificações notáveis durante a erupção da dentição decídua, sendo de extrema importância o conhecimento destes determinantes para a elaboração do diagnóstico.

Palavras chave: Erupção dentária. Dente decíduo. Amamentação. Estado nutricional. Odontopediatria

\begin{abstract}
The purpose of this review of the literature was to investigate the factors that influence chronology of the eruption of the deciduous teeth. A literature survey was done in PubMed and Google Scholar, considering the period from January 1956 to December 2010. It was used as descriptors "nutritional status and eruption of deciduous teeth," changes in the eruption of deciduous teeth, "malnutrition and eruption of deciduous teeth, premature birth and eruption

\footnotetext{
${ }^{1}$ Acadêmica do curso de graduação em Odontologia, Universidade Federal dos Vales do Jequitinhonha e Mucuri, Diamantina, Minas Gerais, Brasil. - mariaeqd_col@yahoo.com.br

${ }^{2}$ Acadêmica do curso de graduação em Odontologia, Universidade Federal dos Vales do Jequitinhonha e Mucuri, Diamantina, Minas Gerais, Brasil.- mayraodontologia@gmail.com Gerais, Brasil- patrícia.faria08@yahoo.com.br

${ }^{4} \mathrm{PhD}$, Professor de Ortodontia, Universidade do Vale do Rio Verde, Departamento de Ortodontia, Três Corações, Minas Gerais, Brasil - lsmarques.prof@gmail.com

${ }^{5} \mathrm{PhD}$, Professora Adjunto de Odontopediatria, Universidade Federal dos Vales do Jequitinhonha e Mucuri, Diamantina, Minas Gerais, Brasil - mlramosjorge@gmail.com
}

${ }^{3}$ Mestranda em Odontopediatria, Universidade Federal dos Vales do Jequitinhonha e Mucuri, Diamantina, Minas
\end{abstract}


of deciduous teeth." Adopted as limit journals published in English language and the total number of articles found $(n=1482), 30$ were read in their entirety because they are directly related to the subject. It was observed in the literature that the eruption of deciduous teeth is influenced by factors such as child nutrition, premature birth, breastfeeding, systemic conditions such as Down syndrome, socioeconomic status, gender, and maternal conditions during pregnancy. The relationship between these factors promotes remarkable changes during the eruption of deciduous teeth, being of extreme importance the knowledge of these determinants for establishing the diagnosis.

Keywords: Tooth eruption. Deciduous teeth. Breastfeeding. Nutritional status. Pediatric Dentistry.

\section{Introdução}

A erupção dentária compreende uma série de eventos que culminam com o aparecimento da coroa dentária no rebordo gengival. Durante a erupção, o dente migra da sua posição intra óssea na maxila e mandíbula até sua posição funcional, ou seja, até entrar em oclusão. Este processo representa parte do crescimento e desenvolvimento infantil e a cronologia de erupção é um indicador de uma série de ocorrências biológicas influenciadas por fatores genéticos e ambientais (ENWONWU, 1973, HOLMAN; YAMAGUCHI, 2005, SINGH et al., 2000; HADDAD; CORREA, 2005; FOLAYAN et al., 2007, OZIEGBE et al., BASTOS et al., 2007).

Estudos realizados em diferentes populações verificaram que o irrompimento dos dentes decíduos tem início entre quatro e 10 meses de idade. A erupção de todos os dentes decíduos ocorre até os 30 meses de vida da criança (FOLAYAN et al., 2007, ASHLEY, 2001, PATRIANOVA et al., 2010).

O momento da erupção dos dentes decíduos não é bem determinado. Estudos têm demonstrado que a cronologia de erupção pode variar entre as populações devido, especialmente, a fatores relativos ao gênero, etnia, alterações sistêmicas, aspectos socioeconômicos, estado nutricional da criança e prematuridade (ENWONWU, 1973, HOLMAN; YAMAGUCHI, 2005, SINGH et al., 2000; HADDAD; CORREA, 2005; FOLAYAN et al.,2007, BASTOS et al., 2007, ALJASER; BELLO, 2003, PSOTER et al., 2008). Porém, poucas evidências existem em relação à real influência desses fatores na alteração da cronologia da erupção dentária.

Portanto, o objetivo do presente estudo foi revisar os fatores associados à cronologia de erupção dos dentes decíduos. 
Métodos

A busca bibliográfica foi conduzida na base de dados PubMed, Lilacs e Google Acadêmico considerando-se o período de 1956 a dezembro de 2010. Os seguintes descritores foram utilizados: "nutritional status and eruption of deciduous teeth", "changes in the eruption of deciduous teeth", "malnutrition and eruption of deciduous teeth", "premature birth and eruption of deciduous teeth", sendo recuperados 1482 artigos. Assim, adotou-se como limite: periódicos publicados nas línguas inglesa e portuguesa. Com os descritores pesquisados, alguns estudos se repetiam e foram eliminadas as repetições, ficando apenas um exemplar de cada artigo. Do total de artigos encontrados, 30 foram lidos na íntegra por estarem diretamente ligados ao tema, sendo excluídos 1442, por não estarem disponíveis na íntegra, estarem repetidos ou não estarem diretamente ligados ao tema.

\section{Resultados}

\section{Cronologia da erupção dentária}

Diversos autores têm investigado as diferenças no momento de erupção dos dentes decíduos em diferentes populações. Em países em desenvolvimento ou subdesenvolvidos este processo tem início entre o nono e décimo mês de vida (YAN et al., 2001, FOLAYAN et al., 2007, PATRIANOVA et al., 2010,).

Estudo realizado com crianças negras do Senegal verificou a seguinte seqüência e cronologia de erupção: incisivo central (9 a 10 meses), incisivo lateral (11 a 12 meses), primeiro molar (15 a 16 meses), caninos (17 a 18 meses) e segundos molares (21 a 28 meses) (YAN et al., 2001). Nas crianças nigerianas, a erupção dentária tem início no oitavo mês de vida com o irrompimento dos incisivos centrais inferiores e a dentição está completa aproximadamente no $25^{\circ}$ mês com a erupção dos segundos molares inferiores (FOLAYAN et al., 2007).

No Brasil, se acordo com um estudo realizado por Patrianova et al. (2010) com 1279 crianças do município de Itajaí, Santa Catarina, os dentes decíduos irrompem entre 11 e 30 meses nas crianças do gênero masculino e entre 12 e 30 meses nas meninas.

Vários fatores podem estar associados a essas diferenças, dentre eles destacam-se o nascimento prematuro, amamentação, estado nutricional infantil, nível 
socioeconômico, condições sistêmicas da criança, gênero, etnia, suplementação nutricional materna durante a gestação e fatores genéticos.

\section{Erupção Dentária e Nascimento Prematuro}

Atrasos na erupção dentária decídua podem estar relacionados à prematuridade ao nascimento. $\mathrm{O}$ nascimento é considerado prematuro quando antecede a trigésima sétima semana de gestação e a criança pode apresentar baixo peso e altura além de comprometimento durante o seu crescimento e desenvolvimento (AKTOREN et al., 2010). Em estudo realizado por Drummond et al. (1992) o atraso na erupção foi observado em 60 a $67 \%$ dos casos quando a comparação foi realizada entre crianças prematuras e crianças nascidas a termo.

Aktoren et al. (2010) acompanharam 178 crianças, de 6 a 39 meses de idade, para avaliar a relação entre a prematuridade e a erupção dos primeiros dentes decíduos. Observou-se que o tempo médio para o surgimento do primeiro dente em lactantes com idade gestacional inferior a 34 semanas, entre 34 e 37 semanas e superior a 37 semanas, foi, respectivamente, $8,8.29$ e 6.93 meses de idade. Uma diferença significativa foi encontrada no tempo de erupção final dos dentes entre os grupos. A erupção tardia foi 3,67 vezes maior em bebês cuja gestação foi inferior a 37 semanas.

Patrianova et al. (2010) realizaram estudo com 1297 crianças brasileiras. Verificou que os bebês com idade gestacional inferior a nove meses apresentam retardo no início da erupção da dentição decídua. No entanto, nos estágios finais da erupção este atraso diminui lentamente.

Entretanto, outro estudo realizado no Brasil por Caixeta e Corrêa (2005), revelou que $42 \%$ das crianças prematuras apresentaram o primeiro dente erupcionado entre o sexto e décimo mês de vida, $36 \%$ entre o décimo e décimo oitavo mês e $3 \%$ até quinto mês. Não se observou atrasos na erupção, porém o número total de dentes até 36 meses de idade mostrou-se menor comparando-se às demais crianças.

Ramos et al.(2006) observou 146 crianças, com idade entre 5 e 36 meses, no Ambulatório de Pediatria do Hospital Universitário Evangélico de Curitiba, Paraná. Considerando-se a idade cronológica, bebês prematuros apresentaram um atraso no tempo de erupção dos primeiros dentes decíduos 
em relação àqueles nascidos no tempo normal.

A divergência entre os
resultados parece ser devido à consideração do período gestacional na contagem do tempo de erupção, sendo que este tempo é inferior para as crianças prematuras. Sendo assim, crianças nascidas antes do tempo normal apresentam atrasos no processo de desenvolvimento, necessitando de um tempo maior para completar a formação e erupção dos dentes após o nascimento. Considerando-se o tempo de gestação e o período que precede a emergência dos primeiros dentes na cavidade bucal, não há diferenças significativas entre prematuros $\mathrm{e}$ crianças nascidas dentro do tempo considerado normal, ou seja, o somatório desses períodos é coincidente. Portanto, essas crianças podem apresentar atrasos na erupção dos primeiros dentes decíduos devido à prematuridade e não a um atraso do desenvolvimento dental (RAMOS et al., 2006).

\section{Erupção dentária e amamentação}

A amamentação no peito também é relacionada como um dos fatores que alteram o calendário da erupção dentária.
Holman e Yamaguchi (2005) observaram que as crianças que nunca tinham sido amamentadas apresentaram atrasos no surgimento dos incisivos superiores e aparecimento acelerado dos segundos molares superiores.

Entretanto, Folayan et al.(2007) não observaram nenhuma diferença significativa no tempo e na seqüência de erupção dos dentes com base no aleitamento materno.

Patrianova et al. (2010) observou um relativo atraso no tempo de erupção dos dentes decíduos em crianças que foram amamentadas após os seis meses de idade. Ele conclui que, a partir do sexto mês de vida, as crianças devem experimentar uma alimentação que estimule o crescimento e desenvolvimento do sistema estomatognático. O tipo de dieta é de fundamental importância para desencadear o processo de erupção da dentição decídua.

\section{Erupção dentária e estado nutricional infantil}

$$
\begin{aligned}
& \text { Alterações na cronologia de } \\
& \text { erupção decídua podem ser } \\
& \text { influenciadas pelo estado nutricional } \\
& \text { infantil, principalmente pela desnutrição } \\
& \text { energético protéica (TOVERUD, 1956, } \\
& \text { BARRET; BROWN, 1966, SWEENEY; }
\end{aligned}
$$


GUZMAN, 1966, MC GREGOR et al., 1968, ENWONWU, 1973, EL LOZY et al., 1975, RAMI REDDY et al.,1986, ALVAREZ et al., 1988, ALVAREZ et al., 1990, ALVAREZ, 1995, HOLMAN; YAMAGUCHI, 2005). Um episódio de desnutrição durante o primeiro ano de vida é suficiente para ocasionar um atraso significativo na erupção da dentição decídua, mesmo decorridos dois anos da desnutrição (ALVAREZ, 1995). Além disso, eles concluíram que a desnutrição crônica tem maior efeito deletério para o processo eruptivo do que a desnutrição aguda. Crianças que apresentaram um quadro de desnutrição crônica sofreram com um insulto nutricional antes do nascimento, período no qual os dentes ainda estão sendo formados, ocasionando em prejuízos no desenvolvimento de todos os dentes decíduos. Já aquelas inseridas em um quadro de desnutrição aguda, o insulto nutricional ocorreu logo após o nascimento ou durante os seis primeiros meses de vida. Nessa fase, os dentes afetados serão apenas os primeiros e segundos molares (ALVAREZ, 1995).

De acordo com o estudo realizado por Bastos et al., em 1998 e 1999, com 359 crianças da cidade de Pelotas, as crianças menores que 49,0 $\mathrm{cm}$ ao nascer apresentaram 0,5 menos pares de dentes emergidos na cavidade bucal aos 6 meses, quando comparadas àquelas nascidas com altura dento dos padrões de normalidade. Além disso, crianças com altura ao nascer igual ou inferior a 49,0 $\mathrm{cm}$ e déficit de altura para a idade de 6 meses, apresentaram 0,1 e 0,4 menos pares de dentes na cavidade bucal aos 12 meses. Observouse, portanto que as crianças com quadros de desnutrição até os 6 meses de idade e com menor comprimento ao nascer, demonstraram um atraso na erupção dentária.

Holman e Yamaguchi (2005) avaliando 114 crianças japonesas observaram que aquelas que apresentavam nutrição inadequada exibiram um retardo no aparecimento dos primeiros dentes em relação às crianças bem nutridas. Os incisivos superiores foram os menos afetados pelo estado de nutrição inadequada, mostrando um atraso de aproximadamente 1,5 meses em relação a uma boa nutrição, sendo que o tempo de erupção aumentou em 20\%. Os incisivos inferiores apresentaram um atraso de 2 meses, aumentando o tempo de erupção em relação a uma nutrição adequada em 26-29\%. A erupção dos caninos e molares foi adiada por 2,7-3,9 meses para crianças com baixa nutrição, 
refletindo $14-21 \%$ de aumento no tempo de emergência.

Um estudo realizado por Delgado et al.(1975), com 273 crianças residentes na zona rural da Guatemala, a partir do final do ano de 1968 a maio 1972, demonstrou que as crianças entre 9 e 15 meses de idade, que nasceram com um peso superior a $3 \mathrm{Kg}$, geralmente apresentavam avanços na erupção dentária quando comparadas com aquelas da mesma idade que nasceram com um peso menor ou igual de $3 \mathrm{Kg}$. Eles observaram também que as crianças agrupadas em um mesmo peso ao nascer, mas que se apresentaram mais pesadas aos 9 e 15 meses de idade, possuíam maior número de dentes erupcionados. Isso sugere um importante efeito do crescimento pósnatal na dentição decídua.

\section{Erupção dentária e nível socioeconômico}

Estudos relacionando a cronologia de irrompimento de decíduos ao nível socioeconômico verificaram que as crianças com escassez de recursos financeiros, apresentaram menos dentes erupcionados do que aquelas de maior poder aquisitivo. ${ }^{6-8}$ Resultado similar foi observado por Singh et al.(2000), em um estudo realizado em 2000, observaram que crianças com baixo nível socioeconômico apresentaram de dois a cinco dentes erupcionados a menos que crianças de maior nível socioeconômico.

Estudos realizados por Folayan et al.(2007) demonstraram uma diferença significativa no tempo de erupção dos caninos superiores, que foi muito superior em crianças de alto nível socioeconômico quando comparadas ao grupo com condições econômicas inferiores.

$$
\text { Alvarez et al. (1988) }
$$

descreveram existir uma associação positiva entre atraso na erupção dos dentes decíduos e baixo nível socioeconômico. Ele afirma que as crianças mais pobres são mais freqüentemente afetadas pela desnutrição, o que acarretaria em alterações nos padrões normais do processo eruptivo.

\section{Erupção dentária e condições sistêmicas}

Atrasos na erupção dentária podem mostrar associação positiva com algumas patologias crônicas. Condições sistêmicas como Síndrome de Down, Síndrome de Turner, Displasia 145 
mucopolissacaridose têm como características o atraso no irrompimento da dentição. ${ }^{7,28}$ Um estudo realizado com 255 portadores de Síndrome de Down enfatizou atrasos na erupção de decíduos. Os pacientes do gênero masculino apresentaram erupção tardia de incisivos e caninos, sendo observado no gênero feminino atraso na erupção de incisivos, caninos e molares (ONDARZA et al.1997).

Staufer et al. (2009) analisaram o processo de erupção dentária de dois pacientes com paralisia cerebral. Eles observaram um padrão de erupção atípico, e atrasos na substituição dos dentes de cerca de cinco anos.

\section{Erupção dentária e gênero}

Diversos estudos presentes na literatura têm demonstrado variações no calendário de erupção dos dentes decíduos relacionadas com o gênero. Alguns autores têm apontado um padrão de aparecimento mais cedo no sexo feminino (CHOI; YANG, 2001), enquanto outros têm observado uma tendência de emergência mais precoce no sexo masculino (TANGUAY et al., 1989, PATRIANOVA, 2004, RAMOS et al., 2006, BASTOS et al. 2007, AKTOREN et al., 2010).
Um estudo realizado por Tanguay et al. (1989) com 195 crianças franco-canadenses (113 do sexo masculino e 82 do sexo feminino), no período de 1975 a 1978, demonstrou que nos meninos a emergência dos dentes decíduos foi adiantada 0,88 meses em relação às meninas. Eles descreveram que os dentes decíduos, individualmente, não irrompem antes nos meninos, mas a formação completa da dentição decídua e mais precoce no sexo masculino. Com exceção dos primeiros molares, todos os dentes apresentam diferenças significativas de emergência, no entanto os incisivos centrais superiores são os dentes de maior relevância quando se leva em consideração a distinção entre os sexos.

Patrianova et al. (2010) observaram que crianças do sexo masculino apresentaram início do processo eruptivo mais precocemente do que as do sexo feminino, com uma variação significativa para os dentes anteriores. A partir daí há uma equiparidade entre os sexos ou mesmo um avanço para o gênero feminino. Em seu estudo, o tempo de erupção total foi de 20,30 meses para o sexo masculino e 19,55 meses para o feminino, demonstrando que as meninas concluem o processo eruptivo em média, antes dos meninos. 
Aktoren et al. (2010), em um estudo envolvendo 178 crianças, observaram que o tempo médio de erupção dos primeiros dentes foi de $7,25( \pm 2,47)$ meses para o sexo feminino e 7,07 $( \pm 1,66)$ meses de idade para o sexo masculino. Além disso, quando os dados foram classificados e avaliados quanto aos tempos de erupção antecipado, normal ou retardado, os meninos $(62,5 \%)$ apresentaram maior tendência para a fase de erupção normal do que as meninas $(37,5 \%)$.

Entretanto, outros autores não encontraram diferenças significativas entre gênero e tempo de emergência dos dentes decíduos (MC GREGOR et al., 1968, HOLMAN; YAMAGUCHI, 2005, FOLAYAN et al., 2007).

\section{Erupção dentária e etnia}

O calendário de erupção da dentição decídua também sofre variações de acordo com as variáveis étnicas, ambientais e culturais (TANGUAY et al., 1989, HOLMAN; YAMAGUCHI, 2005, PATRIANOVA et $a l ., 2010)$.

McGregor et al.(1968), em um estudo realizado em quatro aldeias da Gâmbia, observaram que as crianças de até 18 meses de idade apresentavam, em média, menos dentes erupcionados quando comparadas com estudos realizados em crianças americanas e européias. No entanto, eles não observaram um prolongamento do período total de erupção dentária. Eles também associaram esse atraso a uma alta prevalência de bebês nascidos com baixo peso nas aldeias da Gâmbia.

Um estudo realizado por Folayan et al. (2007) comparou a seqüência de erupção dos dentes decíduos de crianças nigerianas do sexo feminino com dados publicados de outros países (Islândia, Iraque, Arábia Saudita e Estados Unidos). Eles observaram que a seqüência de erupção foi idêntica, alterando apenas o tempo de emergência nos grupos de dentes. A cronologia de erupção para os incisivos centrais inferiores das crianças nigerianas é comparável às iraquianas e árabes sauditas, mas é atrasado em relação às crianças islandesas e norteamericanas. Em relação aos caninos superiores e inferiores e segundos molares superiores, a erupção é anterior em islandeses e posteriores em iraquianos, árabes sauditas e norteamericanos, quando comparados com as crianças nigerianas.

Para avaliar as diferenças entre as populações no calendário de erupção, Holman e Yamaguchi (2005) 147 
emergência dos dentes decíduos de crianças japonesas com estudos realizados em crianças do Bangladesh e Java. Em relação às crianças do Bangladesh, as japonesas apresentaram surgimento dos dentes anteriores adiantados em 1,6 a 4,6 meses. Esse padrão é revertido para os molares superiores, onde as crianças japonesas sofreram atrasos de 2 a 4 meses. $\mathrm{O}$ padrão de erupção entre crianças japonesas e javanesas é similar, embora três dentes diferissem significamente em relação ao tempo de emergência. Os incisivos laterais irromperam mais cedo em crianças japonesas, 1,6 meses (superiores) e 4,6 meses (inferiores), enquanto os primeiros molares superiores sofreram atraso de 3,2 meses em relação às crianças javanesas.

\section{Erupção dentária e genética}

A cronologia e seqüência de erupção dental decídua e um processo que apresenta forte influência genética (FOLAYAN et al., 2007, AKTOREN et al., 2010, PATRIANOVA et al., 2010). Pequenas diferenças no tempo total de erupção decídua poderiam ser explicadas pela interferência dos fatores ambientais e culturais, entretanto, as alterações genéticas determinariam variações maiores, o que se pode observar em crianças com doenças sistêmicas. Algo relevante é que, a partir da emergência do primeiro dente, independente da idade, o tempo total do processo eruptivo mantém-se em torno de 18 a 21 meses, evidenciando a influência genética (PATRIANOVA et al., 2010).

\section{Erupção dentária e suplementação nutricional materna}

Atrasos no calendário de erupção da dentição decídua podem estar relacionados com as condições maternas durante o período pré-natal, incluindo a dieta. $\mathrm{O}$ estado nutricional pode ser determinado em termos de suplementação calórica ingerida durante a gestação. Delgado et al. (1975), em um estudo realizado com 273 crianças na zona rural da Guatemala, observaram que em todas as idades, as crianças cuja mãe pertencia ao grupo de alta suplementação calórica durante a gravidez (>20mil Kcal), têm, em média, mais dentes erupcionados do que aqueles nascidos das mães pertencentes ao grupo de baixa suplementação $(<10$ mil Kcal). Foi observada estreita relação entre suplementação materna, peso ao nascer e padrão de erupção decídua. 148 
durante $o$ pré-natal nasciam mais

pesados e, conseqüentemente,

apresentavam mais dentes erupcionados

em todas as idades subseqüentes.

\section{Considerações finais}

\section{Diversos fatores contribuem \\ para alterações na cronologia de erupção da dentição decídua. O padrão de erupção dentária varia para cada indivíduo, mas existem variáveis que corroboram para o retardo ou precocidade de tal evento. Desvios no processo de normalidade podem estar relacionados com o estado nutricional infantil, nascimento prematuro, amamentação, condições sistêmicas, nível socioeconômico, gênero. Além} desses, fatores inerentes à mãe, tais como a suplementação nutricional durante a gravidez, contribuem significativamente na cronologia de erupção dos dentes decíduos em seus filhos. Pode-se afirmar, portanto, que nenhum fator atua de forma individualizada, sendo notável a interrelação entre os mesmos durante o desenvolvimento da dentição decídua. Existem poucos estudos na literatura que visam correlacionar as alterações no padrão de erupção da dentição decídua com variáveis determinantes para esse processo.

\section{Referências}

1. AKTOREN, O.; TUNA, E.B.; GUVEN, Y.; GOKCAY, G. A study on neonatal factors and eruption time of primary teeth. Community Dent Health., v. 27, n. 1, p. 52-56, mar. 2010.

2. AL-JASER, N.M.; BELLO L.L. Time of eruption of primary dentition in Saudi children. J Contemp Dent Pract., v. 4, n. 3 , p. 65-75, aug. 2003.

3. ALVAREZ, J.O. Nutrition, tooth development and dental caries. Am J Clin Nutr., v. 61, n. 2, p. 410S - 416S, feb., 1995.

4. ALVAREZ, J.O.; EGUREN, J.C.; CACEDA, J.; NAVIA, J.M. The effect of nutritional status on the age distribution of dental caries in the primary teeth. $\mathbf{J}$ Dent Res., v. 69, n. 9, p. 1564 1566, sep., 1990.

5. ALVAREZ, J.O.; LEWIS, C.A.; SAMAN, C.; CACEDA, J.; MONTALVO, J.; FIGUEROA, M.L. Chronic malnutrition, dental caries, and tooth exfoliation in Peruvian children aged 3-9 years. Am J Clin Nutr., v. 48, n. 2, p. 368-372, aug., 1988.

6. ASHLEY, M.P. It's only teething.... report of the myths and modern approaches to teething. Br Dent J., v. 191, n. 1, p. 4-8, jul., 2001.

7. BARRETT, M.J.; BROWN, T. Eruption of deciduous teeth in Australian aborigines. Aust Dent J., v. 11, n. 1, p. 43-50, feb., 1966. 
8. BASTOS, J.L.; PERES, M.A.; PERES, K.G.; BARROS, A.J.

Infant grown, development and tooth emergence patterns: a longitudinal study from birth to 6 years of age. Arch Oral Biol., v. 52, n. 6, p. 598-606, jun., 2007.

9. CAIXETA, F.F.; CORRÊA, M.S. Evaluation of the dental eruption pattern and of enamel defects in the premature child.

Rev Assoc Med Bras., v. 51, n. 4, p. 195-199, jul.-aug., 2005.

10. CHOI, N.K.; YANG, K.H. A study on the eruption timing of primary teeth in Korean children. ASDC J Dent Child., v. 68 , n. 4 , p. 244-249, jul.- aug., 2001.

11. DELGADO, H.; HABICHT, J.P.; YARBROUGH, C.; LECHTIG, A.; MARTORELL, R.; MALINA, R.M.; KLEIN, R.E. Nutritional status and the timing of decíduos tooth eruption. Am J Clin Nutr., v. 28, n. 3, p. 216-224, mar., 1975.

12. DRUMMOND, BK.; RYAN, S.; O'SULIVAN, E.A.; CONDGDON, P.; CURZON, M.E.J. Enamel defects of the primary dentition and prematury. Pediatr Dent., v. 14, n. 2, p. 119-121, mar.-apr., 1992.

13. EL LOZY, M.; REED, R.B.; KERR, G.R.; BOUTOURLINE, E.; TESI, G.; GHAMRY, M.T. et al. Nutritional correlates of child development in southern Tunisia. Growth., v. 39, n. 2, p. 209-221, jun., 1975.
14. ENWONWU, C.O. Influence of socio-economic conditions on dental development in Nigerian children. Arch Oral Biol., v. 18, n. 1, p. 95-107, jan., 1973.

15. FOLAYAN, M.; OWOTABE, F.; ADEJUYIGBE, E.; SEN, S.; LAWAL, B.; NDUKWE, K. The timing of the primary dentition in Nigerian children. Am J Phys Anthropol., v. 134, n. 4, p. 443448, dec., 2007.

16. HADDAD, A.E.; CORREA, M.S. The relationship between the number of erupted primary teeth and the child's height and weight: a cross-sectional study. J Clin Pediatr Dent., v. 29, n. 4, p. 3357-362, summer, 2005.

17. HOLMAN, D.J.; YAMAGUCHI, K. Longitudinal analysis of deciduous tooth emergence: IV - Covariate effects in Japanese children. Am J Phys Anthropol., v. 126, n. 3, p. 352-358, mar., 2005.

18. MC GREGOR, I.A.; THOMSON, A.M.; BILLEWICZ, W.Z. The development of primary teeth in children from a group of Gambian villages, and critical examination of its use or estimating age. Br J Nutr., v. 22, n. 2, p. 307-314, may., 1968.

19. ONDARZA, A.; JARA, L.; MUÑOZ, P.; BLANCO, R. Sequence of eruption of deciduous in a Chilean sample with Down's syndrome. Arch Oral Biol., v. 42, n. 5, p. 401406, may., 1997. 
20. OZIEGBE E.O., ADENOYASOFOWORA ,C., FOLAYAN, M.O., ESAN, T.A., OWOTABE, F.J. Relationship between sociodemographic and anthropometrics variables and number of erupted primary teeth in suburban Nigerian children. Matern Child Nutr., v. 5, n. 1, p. 86-92, jan., 2009.

21. PATRIANOVA, M.E.; KROLL, C.D; BÉRZIN, F. Seqüência e cronologia de erupção dos dentes decíduos em crianças do município de Itajaí (SC). Rev

Sul-Bras Odontol., v. 7, n. 4, p. 406-413, oct.-dec., 2004.

22. PSOTER, W.; GEBRIAN, B.; PROPHETE, S.; REID, B.; KATZ, R. Effect of early childhood malnutrition on tooth eruption in Haitian adolescents. Community Dent Oral Epidemiol., v. 36, n. 2, p. 179189, apr., 2008.

23. RAMI REDDY, V.; VIJAYALAKSHMI, P.B.; CHANDRASEKHAR, R.B.

Deciduous tooth emergence and physique of Velema children of Southeastern Andhra Pradesh, India. Acta Odontol Pediatr., v. 7, n. 1, p. 1-5, jun., 1986.

24. RAMOS, S.R., GUGISCH, R.C., FRAIZ, F.C. The influence of gestational age and birth weight of the newborn on tooth eruption. J Appl Oral Sci., v. 14, n. 4, p. 228-232, aug., 2006. primary dentition in Amritsar an surrounding area. J Indian Dental Assoc., v. 71, p. 26, 2000.

26. STAUFER, K.; HAMADEH, S.; GESCH, D. Failure of tooth eruption in two patients with cerebral palsy and bruxism-a 10year follow-up: a case report.

Spec Care Dentist., v. 29, n. 4, p. 169-174, jul.-aug., 2009.

27. SWEENEY, E.A, GUZMAN, $M$. Oral conditions in children from three highland villages in Guatemala. Arch Oral Biol., v. 11, n. 7, p. 687-698, jul.-aug., 1966.

28. TANGUAY, R.; DEMIRJIAN, A., THIBAULT, H.W. Sexual dimorphism in the emergence of the deciduous teeth. J Dent Res., v. 63, n. 1, p. 65-68, jan., 1984.

29. TOVERUD, G. The influence of war and post-war conditions on the teeth of Norwegian school children: eruption of permanent teeth and status of deciduous dentition. Milbank Mem Fund Q., v. 34, n. 4, p. 355-430, oct., 1956.

30. YAM, A.A.; CISSE, D.; TAMBA, A., DIOP, F.; DIAGNE, F.; DIOP, K.; BA, I. Chronology and date of eruption of primary teeth in Senegal. Odontostomatol Trop. 2001; 24: 34-8.

25. SINGH, N.; SHARMA, S., SIKRI, V.; SINGH, P. To study the average age of eruption of 